\title{
Self-similar decay of high Reynolds number Taylor-Couette turbulence
}

\author{
Ruben A. Verschoof, ${ }^{1}$ Sander G. Huisman, ${ }^{1}$ Roeland C. A. van der Veen, ${ }^{1}$ \\ Chao Sun,,$^{2,1, *}$ and Detlef Lohse ${ }^{1,3, \dagger}$ \\ ${ }^{1}$ Department of Applied Physics, MESA+ institute and J. M. Burgers Center for Fluid Dynamics, \\ University of Twente, P.O. Box 217, 7500 AE Enschede, The Netherlands \\ ${ }^{2}$ Center for Combustion Energy and Department of Thermal Engineering, Key Laboratory for Thermal \\ Science and Power Engineering of the Ministry of Education, Tsinghua University, \\ 100084 Beijing, China \\ ${ }^{3}$ Max Planck Institute for Dynamics and Self-Organisation, 37077 Göttingen, Germany \\ (Received 12 January 2016; revised manuscript received 28 April 2016; published 27 October 2016)
}

We study the decay of high-Reynolds-number Taylor-Couette turbulence, i.e., the turbulent flow between two coaxial rotating cylinders. To do so, the rotation of the inner cylinder $\left(\operatorname{Re}_{i}=2 \times 10^{6}\right.$, the outer cylinder is at rest) is stopped within $12 \mathrm{~s}$, thus fully removing the energy input to the system. Using a combination of laser Doppler anemometry and particle image velocimetry measurements, six decay decades of the kinetic energy could be captured. First, in the absence of cylinder rotation, the flow-velocity during the decay does not develop any height dependence in contrast to the well-known Taylor vortex state. Second, the radial profile of the azimuthal velocity is found to be self-similar. Nonetheless, the decay of this wall-bounded inhomogeneous turbulent flow does not follow a strict power law as for decaying turbulent homogeneous isotropic flows, but it is faster, due to the strong viscous drag applied by the bounding walls. We theoretically describe the decay in a quantitative way by taking the effects of additional friction at the walls into account.

DOI: 10.1103/PhysRevFluids.1.062402

Turbulence is a phenomenon far from equilibrium: Turbulent flow is driven in one or the other way by some energy input and at the same time energy is dissipated, predominantly (but not exclusively) at the smaller scales. For statistically stationary turbulence, this balance is reflected in the famous picture of the Richardson-Kolmogorov energy cascade [1,2]. While the driving on large scales clearly is nonuniversal, depending on the flow geometry and stirring mechanism, the energy dissipation mechanism has been hypothesized to be self-similar [3-8].

How exactly is the energy taken out of the system? A good way to find out is to turn off the driving and follow the then decaying turbulence, as then all scales are probed during the decay process. This has been done in various studies in recent decades for homogeneous isotropic turbulence (HIT). Experimentally, the focus of attention was on grid-induced turbulence [8-15], whereas in numerical simulations periodic boundary conditions were used [16-19]. To what degree the decay of the turbulence depends on the initial conditions [20-22] and whether or not it is self-similar has controversially been debated [5,11,16,23-27]. We note that for HIT, already from dimensional analysis one obtains power laws for the temporal evolution of the vorticity and kinetic energy in decaying turbulence, namely, $\omega(t) \propto t^{-3 / 2}$ and $k(t) \propto t^{-2}$, respectively, in good agreement with many measurements $[10,12,28]$. These scaling laws are also obtained [29] when employing the variable range mean field theory of Ref. [30], developed for HIT. In that way, the late-time behavior, when the flow is already viscosity dominated, can also be calculated, allowing for the calculation of the lifetime of the decaying turbulence [29].

\footnotetext{
*chaosun@tsinghua.edu.ch

†d.lohse@utwente.nl
} 


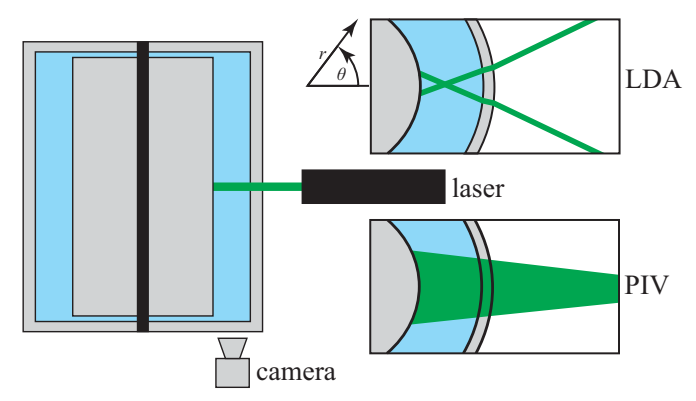

FIG. 1. Schematic of the vertical cross section of the $\mathrm{T}^{3} \mathrm{C}$ facility. The laser beams are in the horizontal plane $(r, \theta)$ at midheight; $z=L / 2$ (unless stated otherwise) for both the LDA and PIV measurements. The top right inset shows the horizontal cross section, showing the LDA beams (not to scale). The beams refract twice on the OC and intersect at the middle of the gap $\left(r=r_{m}\right)$, giving the local velocity component $u_{\theta}$. The bottom right inset shows, for the PIV measurements, particles illuminated by a thin laser sheet. We use the viewing windows in the end plate to look at the flow from the bottom, thus obtaining the velocity components $u_{\theta}$ and $u_{r}$ in the $(r, \theta)$ plane.

However, real turbulence is neither homogeneous nor isotropic, but it has anisotropies and is wall-bounded, with a considerable fraction of the dissipation taking place in the corresponding boundary layers. Studies on the decay of fully developed turbulence flow in wall-bounded flows are, however, scarce [31], though exploring the decay of such flows would teach us about the energy dissipation in the boundary layers and its possible universality. The reason for the scarcity of such studies may be that for the most canonical and best-studied wall-bounded flow, namely, pipe flow [32-35], the decaying turbulent flow is flushed away downstream so that it is hard to study it.

This problem is avoided in confined and at the same time closed turbulent flows, such as RayleighBénard flow [36,37] or Taylor-Couette (TC) flow [38-40], i.e., the flow between two independently rotating coaxial cylinders (Fig. 1). Indeed, turbulent TC flow is neither homogeneous nor isotropic, due to coherent structures that persist also at high Reynolds numbers [41,42], and the boundary layers play the determining role in the angular momentum transfer from the inner to the outer cylinder $[43,44]$.

In this study we employ the TC system to study the temporal and spatial behavior of decaying confined and wall-bounded turbulence and compare it with the known results for HIT, thus complementing the study of Ref. [8] for decaying homogeneous isotropic turbulence. We suddenly stop the inner cylinder rotation (similarly as in Ref. [45], which focused on the decay of turbulent puffs for much lower Reynolds number) and then measure the velocity field over time. We find that the decay models for HIT $[8,29]$ are insufficient to describe the data, but when extending them by explicitly taking the wall friction into consideration, the measured data can be well described. Though the decay does not follow a power law due to the wall friction, the velocity profiles are still self-similar. In the study we restrict ourselves to a fixed outer cylinder and decaying flow; for a numerical study on flow stabilization by a corotating outer cylinder we refer the reader to Ref. [46].

The experiments were performed at the Twente Turbulent Taylor-Couette facility $\left(\mathrm{T}^{3} \mathrm{C}\right)$ [47], consisting of two independently rotating concentric smooth cylinders. The setup has an inner cylinder (IC) with a radius of $r_{i}=200 \mathrm{~mm}$ and an outer cylinder (OC) with a radius of $r_{o}=279 \mathrm{~mm}$, giving a mean radius $r_{m}=\left(r_{i}+r_{o}\right) / 2=239.5 \mathrm{~mm}$, a radius ratio of $\eta=r_{i} / r_{o}=0.716$, and a gap width $d=r_{o}-r_{i}=79 \mathrm{~mm}$. The IC can rotate up to $f_{i}=20 \mathrm{~Hz}$, resulting in a Reynolds number up to $\operatorname{Re}_{i}=2 \pi f_{i} r_{i} d / \nu=2 \times 10^{6}$ with water as the working fluid at $T=20^{\circ} \mathrm{C}$. The cylinders have a height of $L=927 \mathrm{~mm}$, giving an aspect ratio of $\Gamma=L / d=11.7$. The transparent acrylic OC allows for nonintrusive optical measurements. The end plates, which are partly transparent, are fixed to the OC. The velocity is measured using two nonintrusive optical methods: particle image velocimetry (PIV) and laser Doppler anemometry (LDA), as shown in Fig. 1. The LDA measurements give 


\section{DECAY OF TAYLOR-COUETTE TURBULENCE ...}
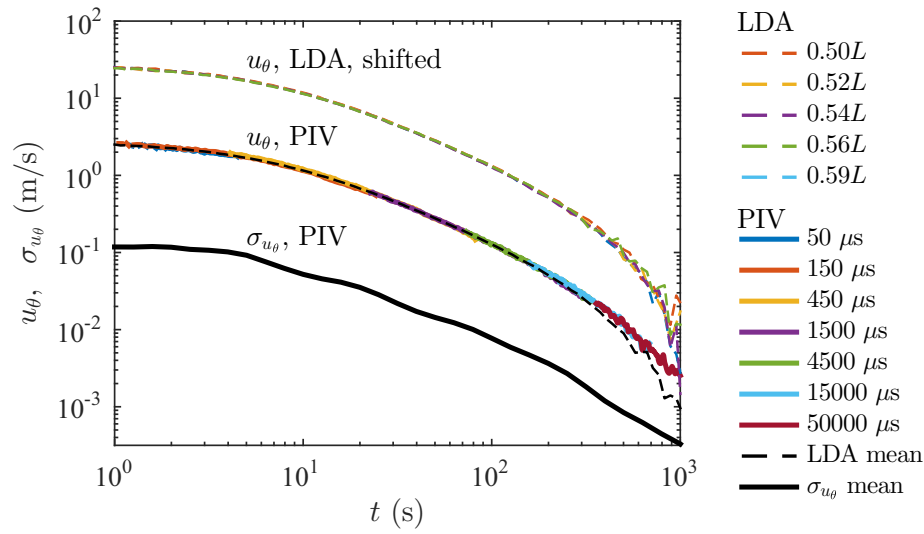

FIG. 2. Midgap azimuthal velocity as a function of time at midheight. PIV measurements with seven different interframe times $\Delta t$ were performed, as shown in the legend, to produce accurate results over the entire velocity range. The PIV measurements are averaged azimuthally and radially; we average over $r_{m}-4 \mathrm{~mm}<r<r_{m}+4 \mathrm{~mm}$, corresponding to $10 \%$ of the gap width. These results are confirmed by LDA measurements performed at several heights (dashed lines), which are shifted by one decade for clarity. The standard deviation $\sigma_{u_{\theta}}$, a measure for the spatial velocity fluctuations, is shown as the solid black line. The data are averaged azimuthally and radially as described above and binned using logarithmic bins of 0.1 decades. The measurements cover three orders of magnitude of the velocity, corresponding to six orders of magnitude in kinetic energy. The measurement uncertainty roughly corresponds to the width of the lines.

the azimuthal velocity $u_{\theta}(t)$ at midgap $\left(r=r_{m}\right)$ and at several heights. The water is seeded with $5-\mu \mathrm{m}$ - diameter polyamide tracer particles with a density of $1.03 \mathrm{~g} / \mathrm{cm}^{3}$. The laser beams are focused in the middle of the gap, i.e., at $r=r_{m}$. Using numerical ray tracing, the curvature effects of the OC are accounted for [48]. The PIV measurements are performed in the $\theta-r$ plane at midheight $(z=L / 2)$, using a high-resolution camera [49], operating at $20 \mathrm{~Hz}$. The spatial resolution of the PIV measurements is $0.04 \mathrm{~mm} /$ pixel, with interrogation windows of 32 pixel $\times 32$ pixel. The flow is illuminated from the side with a pulsed Nd:YLF laser [50], with which a horizontal light sheet is created (Fig. 1). The water is seeded with $20-\mu \mathrm{m}$ polyamide tracer particles. Because of the large velocity range of our measurements, several measurements with a changing $\Delta t$ are performed $(50 \mu \mathrm{s} \leqslant \Delta t \leqslant 50 \mathrm{~ms}$ ) so that the entire velocity range is fully captured. The PIV measurements are processed to give both the radial velocity $u_{r}(\theta, r, t)$ and azimuthal velocity $u_{\theta}(\theta, r, t)$. The Stokes number of the seeding particles are always smaller than $\mathrm{St}=\tau_{p} / \tau_{\eta}<0.2$, so the particles faithfully follow the flow $[2,51]$.

We first drive the turbulence at a rotation rate of $f_{i}=20 \mathrm{~Hz}\left(\mathrm{Re}_{i}=2 \times 10^{6}\right)$ of the IC, while the $\mathrm{OC}$ is at rest, allowing for the development of a statistically stationary state. We then decelerate the IC within approximately $12 \mathrm{~s}$ linearly down to $f_{i}=0 \mathrm{~Hz}$, so, starting from $t=0 \mathrm{~s}$, there is zero energy input. The deceleration rate is limited by the braking power of the electric motor. The deceleration time is much smaller than the typical time scale for turbulence decay $\left(\tau=d^{2} / v \approx 6 \times 10^{3} \mathrm{~s}\right)$. The velocity measurements start when the IC has come to rest, so at $t=0, f_{i}=f_{o}=0 \mathrm{~Hz}$.

In Fig. 2, the azimuthal velocity decay $u_{\theta}(t)$ is shown. The results obtained with PIV and LDA are the same; the LDA results only start to deviate from the PIV measurements when the measured velocities are close to the dynamic range of the LDA system. Viscous friction dissipates the energy, bringing the fluid eventually to rest. Also, the spatial velocity fluctuations, characterized by the standard deviation of the azimuthal velocity fluctuations $\sigma_{u_{\theta}}(t)$, decay in a very similar way (see Figs. 2 and 4). The LDA data (dashed lines shown in Fig. 2) are measured at different heights using LDA; their collapse indicates that during the decay no Taylor rolls develop, which would lead to a height dependence of the profiles. This is in contrast to TC flow with increasing inner 
VERSCHOOF, HUISMAN, VAN DER VEEN, SUN, AND LOHSE
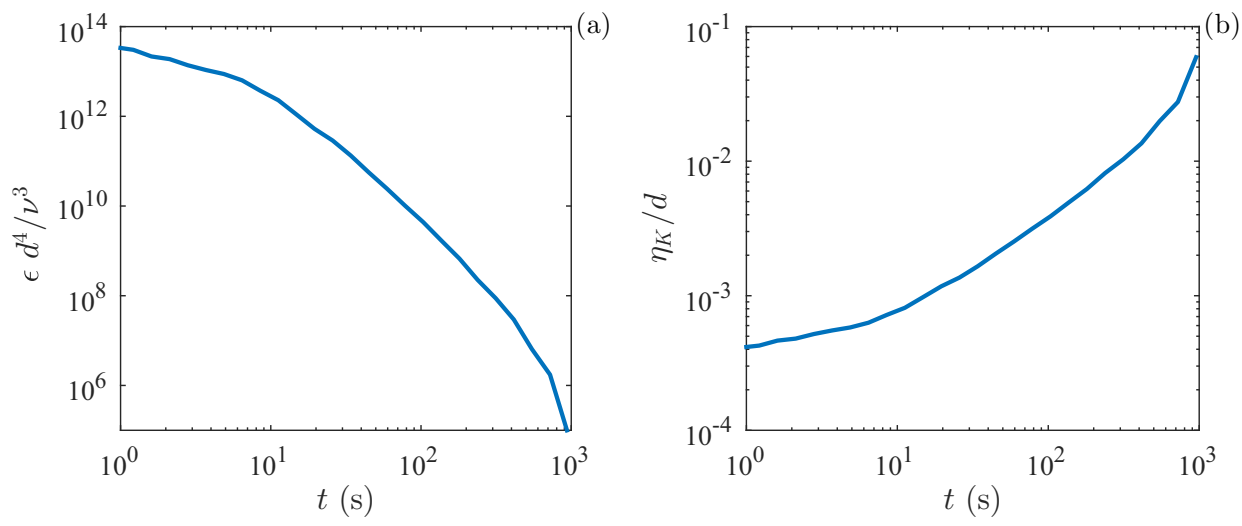

FIG. 3. (a) Energy dissipation rate, normalized with $v^{3} / d^{4}$, as a function of time. Here $\epsilon$ is calculated from the PIV data as shown in Fig. 2; $\epsilon$ drops by more than 8 orders of magnitude. (b) Kolmogorov length scale $\eta_{K}$ as a function of time, normalized with the gap width $d$. As time progresses, the separation of scales becomes smaller, although $\eta_{K}$ remains small.

cylinder rotation, where with increasing $\mathrm{Re}_{i}$ first Taylor rolls develop [52], before one arrives at the structureless fully developed turbulent state (for the chosen geometry) [40,53]. The reason for this difference is that in the constantly rotating case angular momentum is transported from the inner to the outer cylinder, whereas in the decaying case the angular momentum is transported from the bulk to both walls, i.e., a net momentum transport between the cylinders is absent. From earlier work [43] we know that the normalized velocity profiles in the bulk are nearly Re independent and height independent over a large range of Reynolds numbers. Here we focus on the bulk flow velocity where the Re independence holds. Correspondingly, we would get similar results for different $\mathrm{Re}$ measurements in the turbulent regime. The axial and radial velocities are approximately 50-100 times smaller than the azimuthal velocity, so their contributions to the total kinetic energy are negligible.

Turbulence is characterized by a fluid motion over a large range of length scales. In TC flow, the upper limit is the gap width $d$ and the smallest length scale is the Kolmogorov scale $\eta_{K}$, which is defined as $\eta_{K}=\left(v^{3} / \epsilon\right)^{1 / 4}$. From the bulk velocity as measured with PIV, we calculate the energy dissipation rate from the change in velocity over time, i.e., $\epsilon=d\left(\frac{1}{2} u_{\theta}^{2}\right) / d t$. As shown in Fig. 3, as the velocity decreases, also the energy dissipation rate becomes smaller. Clearly, the dissipative length scale changes over time, though $\eta_{K}$ only remains a fraction of the gap. Consequently, we cannot faithfully resolve spatial gradients in the flow with our PIV data.

To compare the experimental data on the decay of the velocity and their fluctuations with theory, we first define the respective Reynolds numbers, namely, $\operatorname{Re}_{u_{\theta}}(t)=u_{\theta}\left(r=r_{m}, t\right) d / v$ taken at midgap $r_{m}$ and $\operatorname{Re}_{\sigma_{\theta}}(t)=$ const $\times \sigma_{u_{\theta}}\left(r=r_{m}, t\right) d / v$ for the fluctuations, which we have rescaled with a constant so that it collapses with $\operatorname{Re}_{u_{\theta}}$ at $t=0$, i.e., const $=\operatorname{Re}_{u_{\theta}}(0) / \sigma_{u_{\theta}}(0) d / v$. The curves show that the decay of the velocity itself and the fluctuations is the same [see Fig. 4(a)]. We then compare the decay of $\operatorname{Re}(t)$ with the one predicted for the theory of HIT, as it follows from a numerical integration of the ordinary differential equation obtained in the model of Ref. [29],

$$
\dot{\mathrm{Re}}=-\frac{1}{3} \frac{v}{d^{2}} c_{\mathrm{HIT}}(\operatorname{Re}) \operatorname{Re}^{2},
$$

with $c_{\mathrm{HIT}}(\mathrm{Re})$ given by Eq. (6) of [29]. From Fig. 4 we see that, though in the beginning the decay is reasonably well described, at a later time the decay experimentally found in this wall-bounded flow is much faster than resulting from the model for HIT. We therefore replace the model for $c_{\mathrm{HIT}}(\mathrm{Re})$ in Eq. (1) by a model for wall-bounded flow, namely, by a friction factor $c_{f}(\mathrm{Re})$ following from the 


\section{DECAY OF TAYLOR-COUETTE TURBULENCE ...}
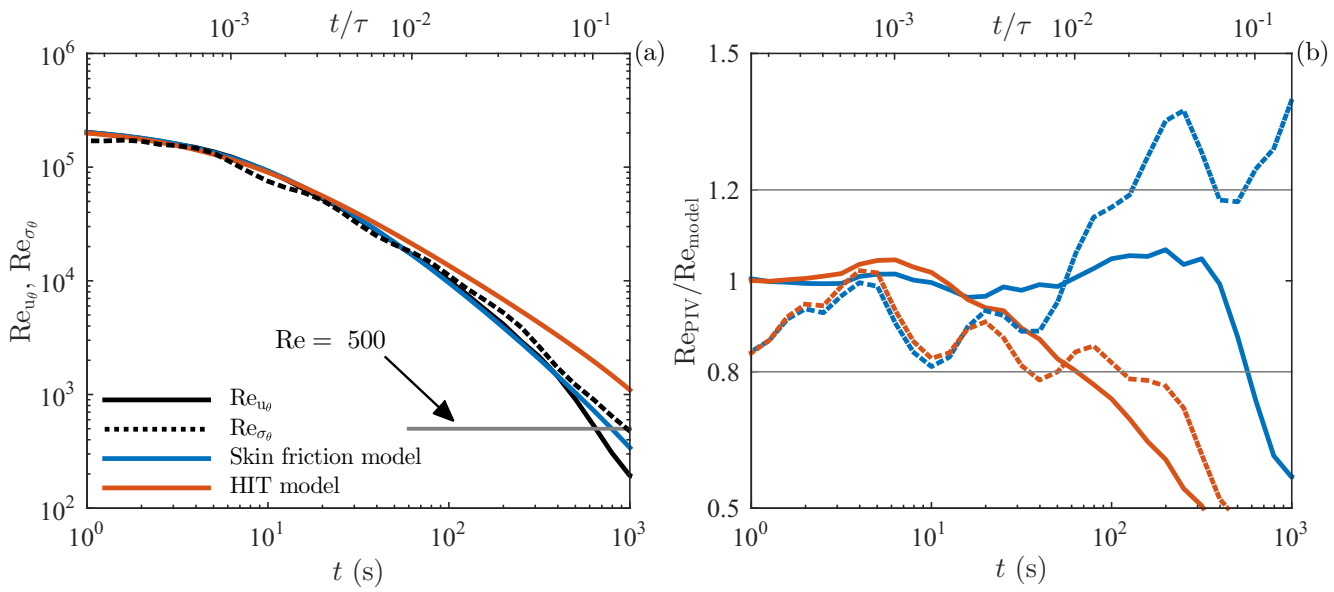

FIG. 4. (a) The PIV results for $\operatorname{Re}_{u_{\theta}}$ and $\operatorname{Re}_{\sigma_{\theta}}$, binned using logarithmic bins of 0.1 decades: The scale on the $y$-axis refers to $\operatorname{Re}_{u_{\theta}}$, whereas the one for $\operatorname{Re}_{\sigma_{\theta}}$ is vertically shifted to show that the decay of the spatial fluctuations and the mean is the same. On the horizontal axis, both the real time and the nondimensionalized time (normalized by $\tau=d^{2} / \nu$ ) are shown. Included in the graph are the results for the HIT model of Ref. [29] and those of the Prandtl-von Kármán skin friction model (2), which includes the effects of the walls. Below the short line at $\operatorname{Re}=500$, thermal effects set in. (b) Ratio between the measurements and, respectively, the HIT model (solid red line) and the skin friction model (solid blue line). The respective dashed lines show the ratio between the fluctuation decay and the two models.

Prandtl-von Kármán skin friction law [2,32,42]

$$
\frac{1}{\sqrt{c_{f}}}=a \log _{10}\left(\operatorname{Re} \sqrt{c_{f}}\right)+b
$$

For pipe flow, a good description of various experimental data can be achieved with $a=1.9$ and $b=-0.3$ [54]. These values depend on the boundary conditions of the flow, i.e., on the geometry and whether or not the flow is actively driven or decaying. For decaying turbulence in the TC geometry we find that a good least-squares fit of this model to the decay of $\operatorname{Re}(t)$ is achieved with $a=2.72$ and $b=-2.22$ (see Fig. 4). As can be seen, due to the extra friction in the wall regions, the decay is now faster than the decay observed in HIT. Now the model agrees with the experimental data for a longer time, reflecting that the no-slip boundary conditions force the fluid to slow down faster.

How long do the respective models for HIT [29] and the Prandtl-van Kármán skin friction hold? We define the beginning of the discrepancy between data and models to be $\left|1-\operatorname{Re}_{\mathrm{PIV}} / \operatorname{Re}_{\text {model }}\right|=$ 0.2 , which is visible as thin gray lines in Fig. 4(b). From this definition, we calculate that the discrepancy between the $\operatorname{Re}_{u_{\theta}}$ and the HIT model starts at $t=45 \mathrm{~s}$ and the one between $\operatorname{Re}_{u_{\theta}}$ and the friction model at $t=530 \mathrm{~s}$.

As was discussed in the Introduction, self-similarity is commonly assumed and observed [5,9] in the decay of HIT flows. The question is whether self-similar flow fields still exist in the decay of inhomogeneous wall-bounded turbulence with a strong shear. By analyzing several instantaneous velocity profiles (see Fig. 5), we found that also for this inhomogeneous turbulence the normalized velocity profiles are self-similar during the decay (see Fig. 6). We find that the normalized velocity profile is self-similar up to $t \approx 400 \mathrm{~s}$. Hitherto, a self-similar decay has not yet been observed for wall-bounded inhomogeneous turbulence, and it is remarkable that also in this highly inhomogeneous and anisotropic flow a self-similar decay exists. Eventually, the self-similarity breaks down, possibly due to thermal convection. Residual cooling in the end plates causes small temperature differences and thus thermal convection is estimated to start from $\mathrm{Re} \approx 500$ (see Ref. [47] for a detailed discussion). Therefore, the results after $t \approx 600 \mathrm{~s}$ are dominated by effects other than the initial 

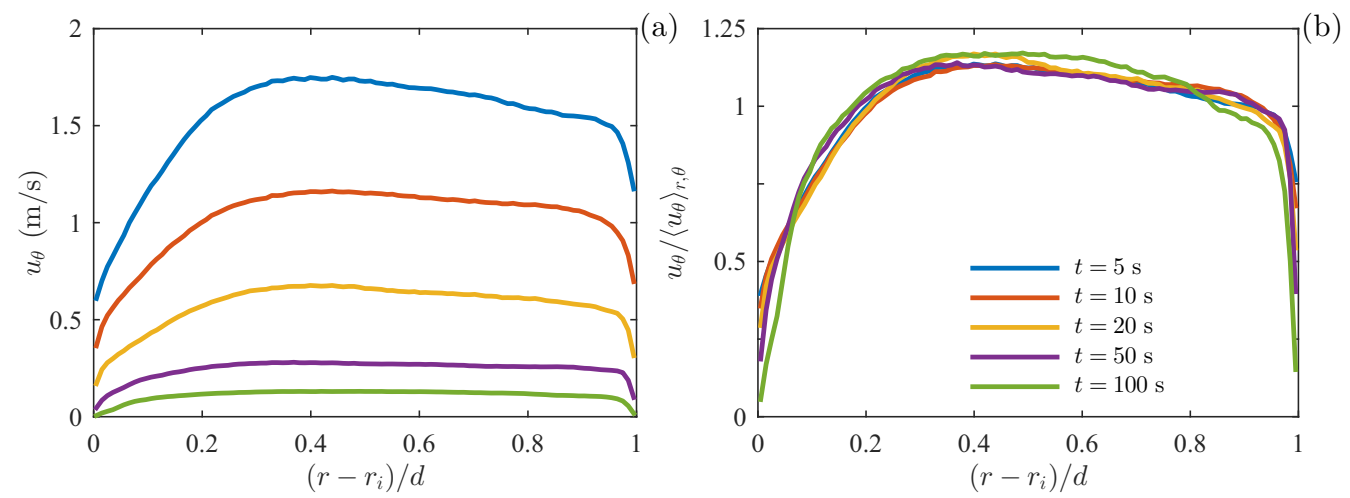

FIG. 5. Azimuthal velocity profiles $u_{\theta}(r)$ for $z=L / 2$, averaged over $\theta$. The decelerating effects of the walls (left and right edges of the figure) can be seen clearly. (b) The velocity is normalized with the (spatial) mean azimuthal velocity $\left\langle u_{\theta}\right\rangle_{r, \theta}(t)$. The normalized velocity profiles overlap, indicating the self-similarity of the velocity profile during the decay.

velocity and the decay process. As can be seen in Fig. 4, this roughly coincides with the moment the model starts to deviate from our measurements.

In conclusion, we measured six decades of the decaying energy in Taylor-Couette flow after the cylinders were halted. During the decay, no height dependence of the flow develops, which is in contrast to the upstarting case, in which the well-known Taylor vortices develop. The azimuthal velocity profile was found to be self-similar. Nonetheless, the kinetic energy in this wall-bounded flow decays faster than observed for homogeneous isotropic turbulent flows. This accelerated decay is due to the additional friction with the walls. We successfully modeled this accelerated decay by using a friction coefficient in which the Prandtl-von Kármán skin friction law for wall-bounded flow is used to model $c_{f}(\mathrm{Re})$. With this model, both the decay of the mean and the fluctuations could be described successfully. We hope that this work will stimulate further investigations into the decay

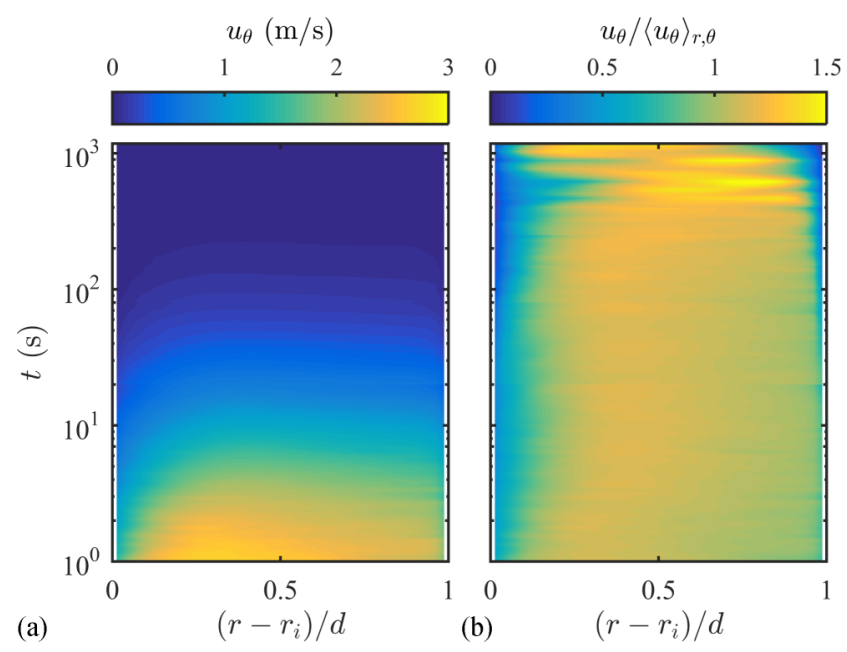

FIG. 6. (a) Measured velocity $u_{\theta}$ as a function of time, resulting from seven PIV measurements with changing $\Delta t$ and averaged over $\theta$. (b) Same results as shown in (a) but now normalized with the mean velocity $\left\langle u_{\theta}(t)\right\rangle_{r, \theta}$. The normalized velocity is self-similar up to $t \approx 400 \mathrm{~s}$. 


\section{DECAY OF TAYLOR-COUETTE TURBULENCE ...}

of wall-bounded (and thus nonisotropic and inhomogeneous) turbulence in other flow geometries, disentangling the universal and nonuniversal features.

The authors gratefully acknowledge informative discussions with and the valuable input of Bruno Eckhardt. We would also like to thank Gert-Wim Bruggert and Martin Bos for their continual technical support over the years and Leen van Wijngaarden, Dennis Bakhuis, and Rodrigo Ezeta Aparicio for various stimulating discussions. This work was supported by the Dutch Foundation for Fundamental Research on Matter FOM, the Dutch Technology Foundation STW, and an ERC grant.

[1] A. N. Kolmogorov, The local structure of turbulence in incompressible viscous fluid for very large Reynolds numbers, Dokl. Akad. Nauk SSSR 30, 301 (1941) [reprinted in Proc. R. Soc. London Ser. A 434, 9 (1991)].

[2] S. B. Pope, Turbulent Flow (Cambridge University Press, Cambridge, 2000).

[3] A. N. Kolmogorov, On the degeneration of isotropic turbulence in an incompressible viscous liquid, Dokl. Akad. Nauk SSSR 31, 319 (1941).

[4] A. N. Kolmogorov, Dissipation of energy in locally isotropic turbulence, Dokl. Akad. Nauk SSSR 32, 16 (1941) [reprinted in Proc. R. Soc. London Ser. A 434, 15 (1991)].

[5] P. G. Saffman, Note on decay of homogeneous turbulence, Phys. Fluids 10, 1349 (1967).

[6] K. R. Sreenivasan, On the scaling of the turbulence energy dissipation rate, Phys. Fluids 27, 1048 (1984).

[7] J. C. Vassilicos, Dissipation in turbulent flows, Annu. Rev. Fluid Mech. 47, 95 (2015).

[8] M. Sinhuber, E. Bodenschatz, and G. P. Bewley, Decay of Turbulence at High Reynolds Numbers, Phys. Rev. Lett. 114, 034501 (2015).

[9] W. K. George, The decay of homogeneous isotropic turbulence, Phys. Fluids A 4, 1492 (1992).

[10] M. R. Smith, R. J. Donnelly, N. Goldenfeld, and W. F. Vinen, Decay of Vorticity in Homogeneous Turbulence, Phys. Rev. Lett. 71, 2583 (1993).

[11] W. K. George and H. Wang, The exponential decay of homogeneous turbulence, Phys. Fluids 21, 025108 (2009).

[12] S. R. Stalp, L. Skrbek, and R. J. Donnelly, Decay of Grid Turbulence in a Finite Channel, Phys. Rev. Lett. 82, 4831 (1999).

[13] L. Skrbek and S. R. Stalp, On the decay of homogeneous isotropic turbulence, Phys. Fluids 12, 1997 (2000).

[14] P. Burattini, P. Lavoie, and R. A. Antonia, On the normalized turbulent energy dissipation rate, Phys. Fluids 17, 098103 (2005).

[15] A. Thormann and C. Meneveau, Decay of homogeneous, nearly isotropic turbulence behind active fractal grids, Phys. Fluids 26, 025112 (2014).

[16] L. Biferale, G. Boffetta, A. Celani, A. Lanotte, F. Toschi, and M. Vergassola, The decay of homogeneous anisotropic turbulence, Phys. Fluids 15, 2105 (2003).

[17] P. Burattini, P. Lavoie, A. Agrawal, L. Djenidi, and R. A. Antonia, Power law of decaying homogeneous isotropic turbulence at low Reynolds number, Phys. Rev. E 73, 066304 (2006).

[18] T. Ishida, P. A. Davidson, and Y. Kaneda, On the decay of isotropic turbulence, J. Fluid Mech. 564, 455 (2006).

[19] T. Teitelbaum and P. D. Mininni, Effect of Helicity and Rotation on the Free Decay of Turbulent Flows, Phys. Rev. Lett. 103, 014501 (2009).

[20] P. Lavoie, L. Djenidi, and R. A. Antonia, Effects of initial conditions in decaying turbulence generated by passive grids, J. Fluid Mech. 585, 395 (2007).

[21] P. C. Valente and J. C. Vassilicos, The decay of turbulence generated by a class of multiscale grids, J. Fluid Mech. 687, 300 (2011). 
[22] D. Hurst and J. C. Vassilicos, Scalings and decay of fractal-generated turbulence, Phys. Fluids 19, 035103 (2007).

[23] R. A. Antonia, R. J. Smalley, T. Zhou, F. Anselmet, and L. Danaila, Similarity of energy structure functions in decaying homogeneous isotropic turbulence, J. Fluid Mech. 487, 245 (2003).

[24] G. L. Eyink and D. J. Thompson, Free decay of turbulence and breakdown of self-similarity, Phys. Fluids 12, 477 (2000).

[25] M. Meldi, P. Sagaut, and D. Lucor, A stochastic view of isotropic turbulence decay, J. Fluid Mech. 668, 351 (2011).

[26] G. Riboux, F. Risso, and D. Legendre, Experimental characterization of the agitation generated by bubbles rising at high Reynolds number, J. Fluid Mech. 643, 509 (2010).

[27] P. A. Davidson, The minimum energy decay rate in quasi-isotropic grid turbulence, Phys. Fluids 23, 085108 (2011).

[28] L. D. Landau and E. M. Lifshitz, Fluid Mechanics (Pergamon, Oxford, 1987).

[29] D. Lohse, Crossover from High to Low Reynolds Number Turbulence, Phys. Rev. Lett. 73, 3223 (1994).

[30] H. Effinger and S. Grossmann, Static structure function of turbulent flow from the Navier-Stokes equation, Z. Phys. B 66, 289 (1987).

[31] H. Touil, J.-P. Bertoglio, and L. Shao, The decay of turbulence in a bounded domain, J. Turbul. 3, N49 (2002).

[32] A. J. Smits, B. J. McKeon, and I. Marusic, High-Reynolds number wall turbulence, Annu. Rev. Fluid Mech. 43, 353 (2011).

[33] M. Hultmark, M. Vallikivi, S. C. C. Bailey, and A. J. Smits, Turbulent Pipe Flow at Extreme Reynolds Numbers, Phys. Rev. Lett. 108, 094501 (2012).

[34] I. Marusic, B. J. McKeon, P. A. Monkewitz, H. M. Nagib, A. J. Smits, and K. R. Sreenivasan, Wall-bounded turbulent flows at high Reynolds numbers: Recent advances and key issues, Phys. Fluids 22, 065103 (2010).

[35] A. J. Smits and I. Marusic, Wall-bounded turbulence, Phys. Today 66(9), 25 (2013).

[36] G. Ahlers, S. Grossmann, and D. Lohse, Heat transfer and large scale dynamics in turbulent RayleighBénard convection, Rev. Mod. Phys. 81, 503 (2009).

[37] D. Lohse and K.-Q. Xia, Small-scale properties of turbulent Rayleigh-Bénard convection, Annu. Rev. Fluid Mech. 42, 335 (2010).

[38] R. J. Donnelly, Taylor-Couette flow: The early days, Phys. Today 44(11), 32 (1991).

[39] M. A. Fardin, C. Perge, and N. Taberlet, "The hydrogen atom of fluid dynamics"-Introduction to the Taylor-Couette flow for soft matter scientists, Soft Matter 10, 3523 (2014).

[40] S. Grossmann, D. Lohse, and C. Sun, High Reynolds number Taylor-Couette turbulence, Annu. Rev. Fluid Mech. 48, 53 (2016).

[41] S. G. Huisman, R. C. A. van der Veen, C. Sun, and D. Lohse, Multiple states in highly turbulent TaylorCouette flow, Nat. Commun. 5, 3820 (2014).

[42] G. S. Lewis and H. L. Swinney, Velocity structure functions, scaling, and transitions in high-Reynoldsnumber Couette-Taylor flow, Phys. Rev. E 59, 5457 (1999).

[43] S. G. Huisman, S. Scharnowski, C. Cierpka, C. J. Kähler, D. Lohse, and C. Sun, Logarithmic Boundary Layers in Strong Taylor-Couette Turbulence, Phys. Rev. Lett. 110, 264501 (2013).

[44] R. Ostilla-Mónico, E. P. van der Poel, R. Verzicco, S. Grossmann, and D. Lohse, Boundary layer dynamics at the transition between the classical and the ultimate regime of Taylor-Couette flow, Phys. Fluids 26, 015114 (2014).

[45] D. Borrero-Echeverry, M. F. Schatz, and R. Tagg, Transient turbulence in Taylor-Couette flow, Phys. Rev. E 81, 025301(R) (2010).

[46] R. Ostilla-Mónico, R. Verzicco, S. Grossmann, and D. Lohse, Turbulence decay towards the linearly stable regime of Taylor-Couette flow, J. Fluid Mech. 748, R3 (2014).

[47] D. P. M. van Gils, G. W. Bruggert, D. P. Lathrop, C. Sun, and D. Lohse, The Twente turbulent Taylor-Couette $\left(\mathrm{T}^{3} \mathrm{C}\right)$ facility: Strongly turbulent (multi-phase) flow between independently rotating cylinders, Rev. Sci. Instrum. 82, 025105 (2011).

[48] S. G. Huisman, D. P. M. Van Gils, and C. Sun, Applying laser Doppler anemometry inside a Taylor-Couette geometry using a ray-tracer to correct for curvature effects, Eur. J. Mech. B 36, 115 (2012). 


\section{DECAY OF TAYLOR-COUETTE TURBULENCE ...}

[49] pco, pco.edge camera, double frame sCMOS, $2560 \times 2160$ pixel resolution, operated in dual frame mode.

[50] Litron, LDY303HE series, dual-cavity, pulsed Nd:YLF PIV laser system. The sheet thickness was approximately $1 \mathrm{~mm}$.

[51] R. Mei, Velocity fidelity of flow tracer particles, Exp. Fluids 22, 1 (1996).

[52] C. D. Andereck, S. S. Liu, and H. L. Swinney, Flow regimes in a circular Couette system with independently rotating cylinders, J. Fluid Mech. 164, 155 (1986).

[53] D. P. Lathrop, J. Fineberg, and H. L. Swinney, Transition to shear-driven turbulence in Couette-Taylor flow, Phys. Rev. A 46, 6390 (1992).

[54] M. V. Zagarola and A. J. Smits, Mean-flow scaling of turbulent pipe flow, J. Fluid Mech. 373, 33 (1998). 